\title{
Estimation of the LDL subclasses in ischemic stroke as a risk factor in a Chinese population
}

\author{
Ruisheng Duan ${ }^{1 *}$, Wenjun Xue ${ }^{2 \dagger} \mathbb{D}$, Kunpeng Wang ${ }^{3+}$, Nan Yin ${ }^{1}$, Hongyu Hao ${ }^{1}$, Hongshan Chu', Lijun Wang ${ }^{4}$,
} Peng Meng ${ }^{4^{*}}$ and Le Diao ${ }^{4^{*}}$

\begin{abstract}
Background: Acute ischemic stroke (AIS) is one of the leading causes of mortality and long-term disability worldwide. Our study aims to clarify the role of low-density lipoproteins (LDL) subclasses in the occurrence of AIS and develop a risk xprediction model based on these characteristics to identify high-risk people.

Methods: Five hundred and sixty-six patients with AIS and 197 non-AIS controls were included in this study. Serum lipids and other baseline characteristics including fasting blood glucose (GLU), serum creatinine (Scr), and blood pressure were investigated in relation to occurrence of AIS. The LDL subfractions were classified and measured with the Lipoprint System by a polyacrylamide gel electrophoresis technique.

Results: Levels of LDL-3, LDL-4 and LDL-5 subclasses were significantly higher in the AIS group compared to the non-AIS group and lower level of LDL-1 was prevalent in the AIS patients. Consistently, Spearman correlation coefficient demonstrated that sd-demonevels, especially LDL-3 and LDL-4 levels, were significantly positively correlated with AIS. Furthermore, there is a significant positive correlation between small dense LDL (sd-LDL, that is LDL-3 to 7) levels and serum lipids including total cholesterol (TC), Low density lipoprotein cholesterol (LDL-C), and Triglyceride (TG). Increased LDL-3 and LDL-4 as well as decreased LDL-1 and LDL-2 were correlated to the occurrence of AIS, even in the people with normal LDL-C levels. A new prediction model including 12 variables can accurately predict the AIS risk in Chinese patients (AUC $=0.82 \pm 0.04$ ).

Conclusions: Levels of LDL subclasses should be considered in addition to serum LDL-C in assessment and management of AIS. A new prediction model based on clinical variables including LDL subtractions can help clinicians identify high of AIS, even in the people with norm.
\end{abstract}

Keywords: LDL subclasses, Small dense LDL, Ischemic stroke, Cerebrovascular disease

\footnotetext{
*Correspondence: 13832140261@139.com; mengpeng@biotecan.com; diaole@biotecan.com

${ }^{\dagger}$ Ruisheng Duan, Wenjun Xue and Kunpeng Wang contributed equally to this work.

'Department of Neurology, Hebei General Hospital, Shijiazhuang 050000, Hebei, China

${ }^{4}$ Department of Medicine, Shanghai Zhangjiang institute of Medical innovation, Biotecan Pharmaceuticals co., Itd., Shanghai 201204, China Full list of author information is available at the end of the article
}

(C) The Author(s). 2020 Open Access This article is licensed under a Creative Commons Attribution 4.0 International License, which permits use, sharing, adaptation, distribution and reproduction in any medium or format, as long as you give appropriate credit to the original author(s) and the source, provide a link to the Creative Commons licence, and indicate if changes were made. The images or other third party material in this article are included in the article's Creative Commons licence, unless indicated otherwise in a credit line to the material. If material is not included in the article's Creative Commons licence and your intended use is not permitted by statutory regulation or exceeds the permitted use, you will need to obtain permission directly from the copyright holder. To view a copy of this licence, visit http://creativecommons.org/licenses/by/4.0/ The Creative Commons Public Domain Dedication waiver (http://creativecommons.org/publicdomain/zero/1.0/) applies to the data made available in this article, unless otherwise stated in a credit line to the data. 


\section{Background}

Stroke is known as an acute cerebrovascular disorder with a sudden loss or deterioration of brain function, resulting from ischemic infarction or intracranial hemorrhage. Acute ischemic stroke (AIS) is one of the leading causes of mortality and long-term disability worldwide, which accounts for approximately $80 \%$ of all strokes. According to the Global Burden of Disease (GBD) Study 2016 estimates of stroke Incidence, China had the greatest estimated lifetime risk and bore the biggest stroke burden in the world. Meanwhile, the stroke burden in China has been increasing over the past 30 years, and remains specifically high in rural areas, with the bulk of this burden occurring in the northern and central regions [1].

As a multifactorial disease, stroke occurs due to various genetic and environmental factors like hypertension, smoking, and alcohol use [2,3]. One such vital risk factor responsible for the development of stroke is atherosclerosis (AS) of the cerebral circulation, which is closely associated with the abnormalities of serum lipids and lipoproteins, including elevated triglyceride levels, reduced high-density lipoproteins (HDL) cholesterol concentrations and increased low-density lipoproteins (LDL) cholesterol concentrations [4]. Specifically, LDL comprises multiple distinct subclasses, defined as LDL-1 to LDL-7 in gradient gel electrophoresis, which differ in size, density, physicochemical composition, metabolic behavior and atherogenicity. Among them, LDL-3 to 7 is also named as small dense LDL (sd-LDL) according to their size and density, whereas LDL- 1 and 2 are defined as large LDL correspondingly [4, 5].

According to the National Cholesterol Education Program Adult Treatment Panel III (NCEP III), high level of sd-LDL is an established risk factor for cardiovascular disease (CVD) [6], however, their clinical significance for AIS is underappreciated compared to CVD. Besides, considering the complex etiology of stroke, studies that investigate the association of various risk factors and AIS should be conducted in populations with different genetic and environmental backgrounds. Here, we evaluated serum lipid profile and LDL subtractions in AIS patients and non-AIS controls, analyzed the correlation of clinical characteristics and AIS risk in a Chinese population from northern and central regions, and established a predictive model for AIS risk with selected variances.

\section{Methods}

\section{Study population}

The study group consisted of 566 hospitalized patients (318 males and 248 females; mean age, 66.1 \pm 13.1 years) with the first onset of AIS. The patients were recruited from department of neurology in Hebei General Hospital and the First People's Hospital of
Pingdingshan and department of Neurosurgery in the Affiliated Hospital of Chengde Medical University, between December 2016 and March 2018. The diagnosis of ischemic stroke was based on current guidelines, and computed tomography $(\mathrm{CT})$ or magnetic resonance imaging (MRI) were performed by experienced neurologists to confirm the diagnosis of ischemic stroke and to rule out hemorrhagic stroke.

On admission, essential characteristics and risk factors of patients were collected and evaluated, including age, sex, height, weight, smoking, alcohol use, blood pressure and blood glucose (GLU). Patients included in this study did not use any lipid-lowering therapy. Co-morbidities were also evaluated as follows: hypertension (HT), according to a previous diagnosis, or measured systolic blood pressure (SBP) $\geq 140 \mathrm{mmHg}$, and/or diastolic blood pressure (DBP) $\geq 90 \mathrm{mmHg}$; Presence of cardiovascular disease (previous myocardial infarction, coronary artery disease, valvular heart disease, cardiomyopathy or arrhythmia) and diabetes mellitus (DM), according to a previous diagnosis or current medication for diabetes were also recorded.

Corresponding controls $(n=197 ; 91$ males and 106 females; mean age, $64.7 \pm 13.7$ years) were selected based on the absence of neurological diseases as well as the same exclusion criteria as described for the cases in clinic patients with non-stroke neurological disorders.

Every individual was informed about the aims of the study and provided written consent before participation. Written informed consent was obtained from a relative in the case of patients with impaired cognition.

\section{Blood collection and analysis}

Blood samples were collected in EDTA tubes following overnight fasting for at least $12 \mathrm{~h}$, and analyzed by a solid-phase, enzyme-labeled chemiluminescent immunometric assay on Siemens Advia 2400 with the manufacturer's reagents as directed to detect blood GLU, serum creatinine (Scr), Triglyceride (TG), total cholesterol (TC), High density lipoprotein cholesterol (HDL-C), and Low density lipoprotein cholesterol (LDL-C).

\section{Low-density lipoprotein analysis}

Blood samples were also used for LDL subfraction analysis. LDL subgroups were classified and measured with the Lipoprint System (Quantimetrix Corporation, Redondo Beach, CA, USA) according to the manufacturer's instructions as previously described $[7,8]$. The clinical application of this system is approved by the Food and Drug Administration (FDA). This system separates LDL subfractions by a polyacrylamide gel electrophoresis technique and gels were scanned to determine the relative area of each lipoprotein subfraction after electrophoresis. Based on net surface 
charge and size, various stained LDL subfractions are classified according to their mobilities in the gel. By this analysis, LDL was divided into 7 subfractions (LDL-1 to LDL-7).

\section{Statistical analysis}

Statistical analyses were performed using Python software (Version 3.6). Parametric statistics (t-test) were used for normally distributed data. Nonparametric Mann-Whitney $U$ test were used for non-normally distributed data and expressed as median (25, 75\% IQR). Categorical variables were presented by numbers or proportions, and difference in distribution between groups were analyzed by Chi-square or the Fisher's exact test, as appropriate. If the data are not normally distributed, correlation analysis was performed using the Spearman correlation coefficient. The eXtreme gradient boosting (XGBoost) Classifier decision trees model (Xgboost 0.81), Random Forest Classifier, Logistic Regression, AdaBoost Classifier were performed to estimate the stroke risk with variables. The $\mathrm{k}$-fold cross-validation $(\mathrm{k}=5)$ was implemented to determine the performance. Related data were presented as receiver operating characteristics (ROC) curves and area under the curve (AUC). An AUC value $>0.5$ indicated better predictive values; the closer the AUC to 1, the better the model performance.

\section{Results}

\section{Clinical and laboratory characteristics of study} participants

A total of 566 patients were included in the AIS group and 197 age-matched non-AIS patients were set as control group. The baseline characteristics of both study groups are shown in Table 1. The BMI, the proportion of patients with hypertension, diabetes mellitus, metabolic syndrome, and cardiovascular disease, SBP, DBP, Scr, LDL-C, TG, Apolipoprotein A (ApoA), and sd-LDL were significantly higher than those in the control group $(P<0.05)$. Smoking, and alcohol consumption, and GLU, TC, and Apolipoprotein B (ApoB) did not differ between the two groups, while the HDL-C concentration was significantly higher in the control group.

\section{Distribution of LDL subtractions in AIS patients and controls}

As shown in Table 2, levels of LDL-3, LDL-4 and LDL-5 subclasses were significantly higher in the AIS group

Table 1 Clinical and laboratory characteristics in all subjects

\begin{tabular}{|c|c|c|c|}
\hline Characteristics & AIS $(n=566)$ & Controls $(n=197)$ & $P$ value * \\
\hline Age (years) & $66(57,77)$ & $67(51,76)$ & 0.114 \\
\hline $\operatorname{Sex}(M / F)$ & $318 / 248$ & $91 / 106$ & $0.016^{*}$ \\
\hline BMI $\left(\mathrm{kg} / \mathrm{m}^{2}\right)$ & $24.4(22.4,27.4)$ & $23.4(21.4,25.3)$ & $<0.001 *$ \\
\hline Smoking (\%) & 75 (13.3) & $19(9.6)$ & 0.209 \\
\hline Alcohol use (\%) & $78(13.8)$ & $18(9.1)$ & 0.105 \\
\hline H-type hypertension (\%) & $103(18.2)$ & $18(9.1)$ & $0.002 *$ \\
\hline Hypertension (\%) & $430(76.0)$ & $79(40.1)$ & $<0.001 *$ \\
\hline Diabetes mellitus (\%) & $155(27.4)$ & $17(8.6)$ & $<0.001 *$ \\
\hline Metabolic syndrome (\%) & $115(20.3)$ & $27(13.7)$ & $0.043 *$ \\
\hline Cardiovascular disease (\%) & $464(82.0)$ & $83(42.1)$ & $<0.001 *$ \\
\hline SBP $(\mathrm{mmHg})$ & $139.5(127,156)$ & $123(121,140)$ & $<0.001 *$ \\
\hline DBP (mmHg) & $84(77,94)$ & $78(70,87)$ & $<0.001 *$ \\
\hline GLU (mmol/L) & $5.3(4.7,6.2)$ & $5.2(4.7,6.1)$ & 0.343 \\
\hline $\mathrm{SCr}(\mu \mathrm{mol} / \mathrm{L})$ & $69.9(59,83)$ & $63(53,74)$ & $<0.001 *$ \\
\hline $\mathrm{TC}(\mathrm{mmol} / \mathrm{L})$ & $4.2(3.5,5.1)$ & $4.2(3.5,4.9)$ & 0.228 \\
\hline LDL-C (mmol/L) & $2.5(1.9,3.1)$ & $2.3(1.8,2.8)$ & $0.003 *$ \\
\hline $\mathrm{HDL}-\mathrm{C}(\mathrm{mmol} / \mathrm{L})$ & $1.1(0.8,1.3)$ & $1.2(0.9,1.6)$ & $<0.001 *$ \\
\hline TG (mmol/L) & $1.4(1.0,2.1)$ & $1.2(0.9,1.5)$ & $<0.001 *$ \\
\hline ApoA (mmol/L) & $1.2(1.1,1.4)$ & $0.9(0.8,1.2)$ & $<0.001 *$ \\
\hline ApoB (mmol/L) & $0.8(0.6,0.9)$ & $0.8(0.6,0.9)$ & 0.494 \\
\hline sd-LDL (mg/dL) & $7(2,17)$ & $3(1,6)$ & $<0.001 *$ \\
\hline
\end{tabular}

AIS Acute ischemic stroke, M Male, F Female, BMI Body mass index, SBP Systolic blood pressure, DBP Diastolic blood pressure, GLU Fasting blood glucose, Scr Serum creatinine, TC Total cholesterol, $L D L-C$ Low density lipoprotein cholesterol, HDL-C High density lipoprotein cholesterol, $T G$ Triglyceride, ApoA Apolipoprotein A, ApoB Apolipoprotein B, sd-LDL Small dense LDL; ${ }^{*} p<0.05$ 
Table 2 The distribution of LDL subclasses in AIS patients and controls

\begin{tabular}{llll}
\hline LDL subclasses & AIS $(\boldsymbol{n}=\mathbf{5 6 6})$ & Controls $(\boldsymbol{n}=\mathbf{1 9 7})$ & $\boldsymbol{P}$ value * \\
\hline LDL-1 $(\mathrm{mg} / \mathrm{dL})$ & $21.5(15,29)$ & $23(17,30.5)$ & $0.021^{*}$ \\
LDL-2 $(\mathrm{mg} / \mathrm{dL})$ & $18(13,25)$ & $18(13,24)$ & 0.318 \\
LDL-3 $(\mathrm{mg} / \mathrm{dL})$ & $7(2,13)$ & $3(1,6)$ & $<0.001 *$ \\
LDL-4 $(\mathrm{mg} / \mathrm{dL})$ & $0(0,3)$ & $0(0,0)$ & $<0.001^{*}$ \\
LDL-5 $(\mathrm{mg} / \mathrm{dL})$ & $0(0,0)$ & $0(0,0)$ & $0.032 *$ \\
LDL-6 $(\mathrm{mg} / \mathrm{dL})$ & $0(0,0)$ & $0(0,0)$ & 0.117 \\
LDL-7 $(\mathrm{mg} / \mathrm{dL})$ & $0(0,0)$ & $0(0,0)$ & 0.206
\end{tabular}

LDL Low density lipoprotein; ${ }^{*} p<0.05$

compared to the control group $(p<0.05)$. In contrast, LDL-1 was significantly lower in the AIS group, while there was no difference between the AIS and control groups in terms of LDL-2, LDL-6 and LDL-7 $(p>0.05)$.

\section{Correlations of clinical characteristics and AIS risk}

Spearman correlation coefficient was used to assess the correlation between clinical characteristics and AIS risk, and heatmap was drawn accordingly (Fig. 1). It was found that HDL-C was negatively correlated with presence of AIS in the study cohorts $(r=-0.17, P<0.001)$. A significant positive correlation was found between AIS and several clinical and laboratory features including BMI, hypertension, diabetes mellitus, cardiovascular disease, SBP, DBP, LDL-C, TG, ApoA, sd-LDL, LDL-3 and LDL-4 $(\mathrm{r}>0.1, P<0.001)$.

\section{Correlations of LDL subtractions and LDL-C}

We next performed Spearman correlation coefficient to investigate the correlations between LDL-C and its subclasses (Table 3). As a result, there was a significant positive correlation between LDL-C and LDL-1 $(r=0.540$, $p<0.05), \mathrm{LDL}-2(r=0.547, p<0.05), \mathrm{LDL}-3(r=0.343$, $p<0.05)$ and LDL-4 $(r=0.227, p<0.05)$. With regard to the correlation among LDL subtypes, LDL-1 was found positively correlated with LDL-2 $(r=0.485, p<0.05)$, but negatively correlated with the rest LDL subtypes, whereas LDL-2 was positively correlated with LDL-3 $(r=0.611$, $p<0.05)$ and LDL-4 $(r=0.230, p<0.05)$ besides LDL-1, but negatively correlated with LDL-5 to 7 . For the LDL-3 to 7 , so called sd-LDL, there is a positive correlation between each other in most cases except for LDL-3 and LDL-7 $(r=-0.019, p=0.599)$.

\section{Correlation analysis between sd-LDL and serum lipids}

Spearman correlation coefficient showed a significant positive correlation between sd-LDL levels and serum lipids including TC, LDL-C, and TG, as shown in Table 4 and Fig. 2. Sd-LDL was negatively correlated with HDL-C in control group and all subjects, but not in AIS group.

\section{Clinical and laboratory characteristics of study} participants with normal LDL-C levels

In AIS group, the sd-LDL level was significantly higher in the high LDL-C group of patients than in the normal LDL-C group $(P<0.001)$ (Table 5). Additionally, although there was no significant difference in GLU and LDL-C levels between the AIS group with normal LDL$\mathrm{C}$ levels and the control group $(P>0.05)$, the other laboratory characteristics, including blood pressure, Scr, serum lipids, and LDL subclasses, differed significantly between the two groups, as shown in Table 6 .

\section{Prediction of AIS risk by laboratory characteristics with an established model}

Considering the correlations of laboratory characteristics with AIS, a risk prediction model was established accordingly to identify people most likely to develop AIS (Fig. 3). Several typical and recent artificial intelligence (AI) algorithms were assessed in the context of AIS, and the XGBoost stood out as our base model due to its high performance $(\mathrm{AUC}=0.82 \pm 0.04$ ) (Fig. 3a). As a frequently used resampling method, kfold cross-validation was employed to estimate the performance of the XGBoost classifier (Fig. 3b). The new prediction model contained 12 variables (ie, Scr, SBP, DBP, TG, TC, LDL-C, HDL-C, LDL-1, LDL-2, LDL-3, LDL-4, LDL-5) with a cut-off value of 0.5.

\section{Discussions}

In China, stroke was still the top leading cause of years of life lost (YLLs) and disability-adjusted life-years (DALYs) in 2017 [9]. Various risk factors have been implicated with stoke, and one of the most important traditional risk factors is the elevation of LDL-C, consisting of 7 subtractions, defined as LDL-1 to LDL-7. Existing data on the relationship between LDL subclasses and AIS are however equivocal. The objective of this study was to examine the contribution of LDL subtractions to AIS as a risk factor in a Chinese population and to develop a risk prediction model based on these characteristics to identify people most likely to develop AIS.

The mechanisms related to the atherogenicity of sdLDL have long been recognized, such as penetrating the arterial wall more easily, being more susceptible to be oxidized or binding to the glycosaminoglycans in the arterial wall more readily, and so on, thus it has been stated in several studies as an independent and more potent risk factor for coronary and peripheral artery disease as well as carotid atherosclerosis, compared to LDL-C [10-12]. More recent study in a Japanese population of 345 men $\geq 65$ years old with stable coronary artery disease showed that small dense Low density lipoprotein cholesterol (sdLDL-C) is a more effective secondary biomarker for cardiovascular events than 


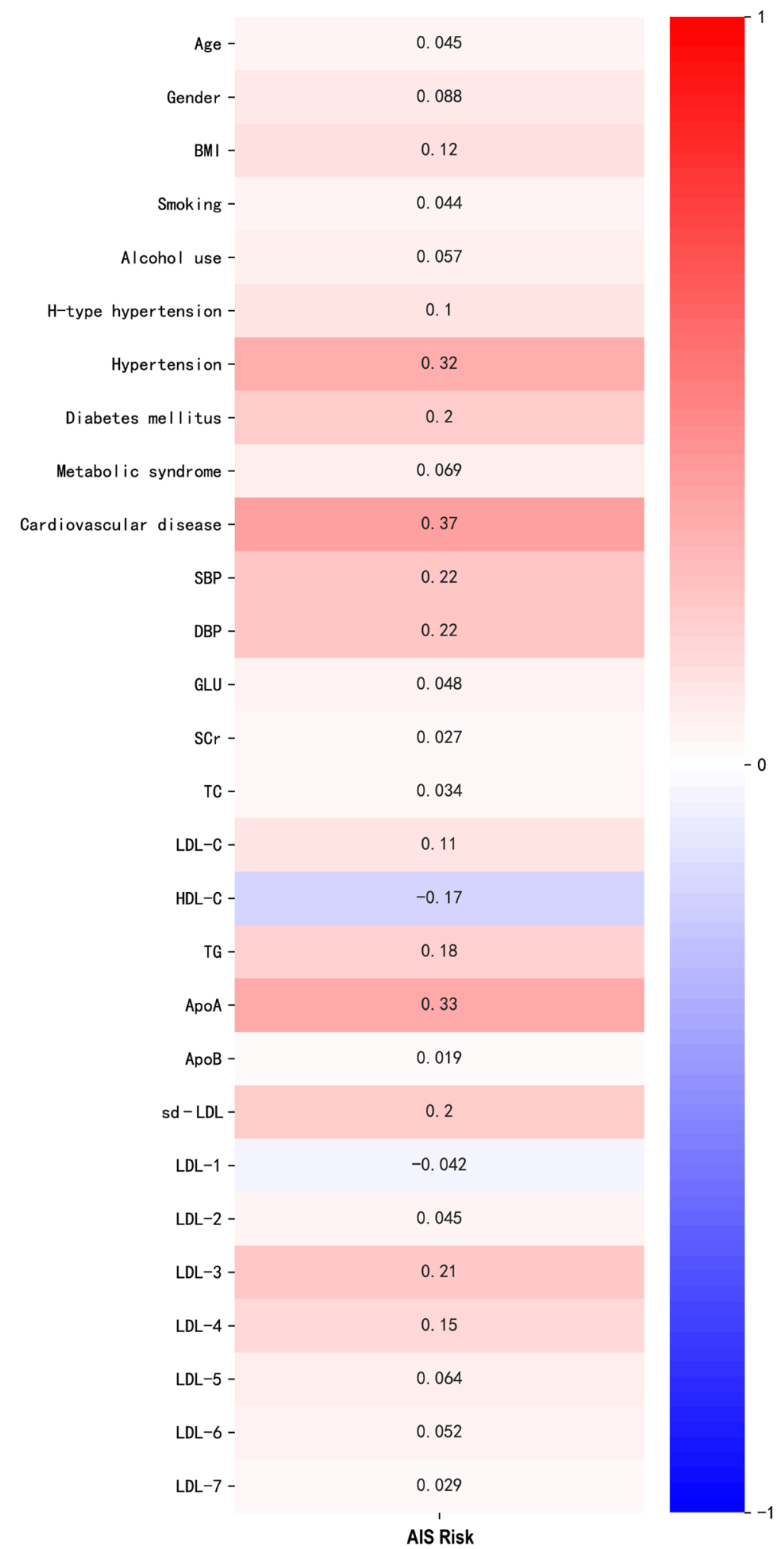

Fig. 1 Heatmap showing the correlation between clinical characteristics and AIS risk 
Table 3 Correlation analysis of $L D L-C$ and its subclasses by Spearman correlation method

\begin{tabular}{|c|c|c|c|c|c|c|c|c|}
\hline & LDL-C & LDL-1 & LDL-2 & LDL-3 & LDL-4 & LDL-5 & LDL-6 & LDL-7 \\
\hline LDL-1 & $0.540^{*}$ & & $0.485^{*}$ & $-0.111^{*}$ & $-0.279^{*}$ & $-0.260^{*}$ & $-0.181^{*}$ & -0.056 \\
\hline LDL-2 & $0.547^{*}$ & & & $0.611^{*}$ & $0.230^{*}$ & -0.066 & $-0.155^{*}$ & $-0.077^{*}$ \\
\hline LDL-3 & $0.343^{*}$ & & & & $0.722^{*}$ & $0.325^{*}$ & $0.089^{*}$ & -0.019 \\
\hline LDL-4 & $0.227^{*}$ & & & & & $0.543^{*}$ & $0.247^{*}$ & 0.057 \\
\hline LDL-5 & 0.063 & & & & & & $0.495^{*}$ & $0.163^{*}$ \\
\hline LDL-6 & -0.046 & & & & & & & $0.345^{*}$ \\
\hline LDL-7 & -0.038 & & & & & & & \\
\hline
\end{tabular}

${ }^{*} p<0.05$

LDL-C [13]. Another study compared the values of six LDL-related variables for predicting the severity of coronary artery disease (CAD), using untreated Chinese patients undergoing coronary angiography (CAG). The predicting value of CAD turned out to be sd-LDL $>$ oxidized-low density lipoprotein (ox-LDL) $>$ ApoB $>$ nonHDL-C $>$ LDL-C $>$ lipoprotein (a) and all variables existed as independent risk factors for the severity of CAD in multivariate logistic analysis [14]. Chaudhary et al. showed that elevated LDL-4 level is an independent predictor of severe CAD (AUC $=0.62, p<0.0001$ ) with a cut-off value of $>16.9 \mathrm{mg} / \mathrm{dL}$, a sensitivity of $53 \%$, and specificity of $79 \%$, respectively [15].

To date, the studies on the relationship between LDL subtractions and AIS are still limited. In this study, we found a significant relationship between sd-LDL and AIS. Sd-LDL was significantly higher in the AIS group than those in the control group and Spearman correlation coefficient showed that sd-LDL levels, particularly LDL-3 and LDL-4 levels, were significantly positively correlated with AIS. Besides, LDL-3, LDL-4 and LDL-5 levels were significantly higher in the AIS group compared to the control group, while LDL-1 was significantly lower. In terms of certain LDL particles, our findings are different from the other two studies by Zeljkovic et al. and Kayran et al. The former Serbia group examined LDL subclasses in 200 AIS patients as well as 162 controls, showing that AIS patients had ignificantly more LDL III and IVb subclasses (sd-LDL), but less large LDL I and II particles [16]. The latter, however, recruited 110 AIS patients and 60 healthy controls from Turkey in the study, and reported that apart from LDL-3 and LDL-4, the level of large LDL-2 particles was also significantly higher in AIS patients [17]. The distribution of large LDLs (LDL-1 and LDL-2) between AIS and control group is in controversy though, all three studies demonstrated a significant elevation of sd-LDLs, especially LDL-3 and LDL-4 levels in the AIS patients.

Additionally, the correlation of LDL subtractions among each other and the relationship of sd-LDL and serum lipids were assessed in our study. Consistent with the lower distribution of LDL-1 in AIS group, LDL-1 was found negatively correlated with the sd-LDL particles, LDL-3 to 7, suggesting lower potential atherogenicity of this particle. By contrast, LDL-2 was positively correlated with LDL-3 and LDL-4, the main atherogenic particles of sd-LDL, which explains the slightly higher distribution of LDL-2 in AIS group and is consistent with the findings by Kayran et al. Considering the correlation coefficients of LDL-1 and LDL-2 with LDL-C, it is highly recommended that not only LDL-C level but also the LDL subtractions should be taken into consideration for the management of AIS. Although our data suggest the lower atherogenicity of LDL-1, other in vivo studies indicate that LDL-1 is more atherogenic than LDL-2, due to its longer half-life, which renders the particle more susceptible to uptake by the scavenger LDL receptor on macrophages [18]. In this case, further studies with larger sample sizes are needed to clarify the function of each LDL subtraction, especially LDL-1 and LDL-2, in AIS development.

Sd-LDL is often accompanied by increased TG, ApoB and decreased HDL levels [10]. Multiple linear regressions

Table 4 Correlation coefficient between sdLDL-C and serum lipids by Spearman correlation method

\begin{tabular}{llll}
\hline Serum lipids & All subjects $(\boldsymbol{n}=\mathbf{7 6 3})$ & AIS $(\boldsymbol{n}=\mathbf{5 6 6})$ & Controls $(\boldsymbol{n}=\mathbf{1 9 7 )}$ \\
\hline TC & $0.315^{*}$ & $0.402^{*}$ & 0.037 \\
LDL-C & $0.310^{*}$ & $0.345^{*}$ & $0.159 *$ \\
HDL-C & $-0.138^{*}$ & 0.067 & $-0.216 *$ \\
TG & $0.536^{*}$ & $0.593 *$ & $0.221 *$
\end{tabular}

${ }^{*} p<0.05$ 

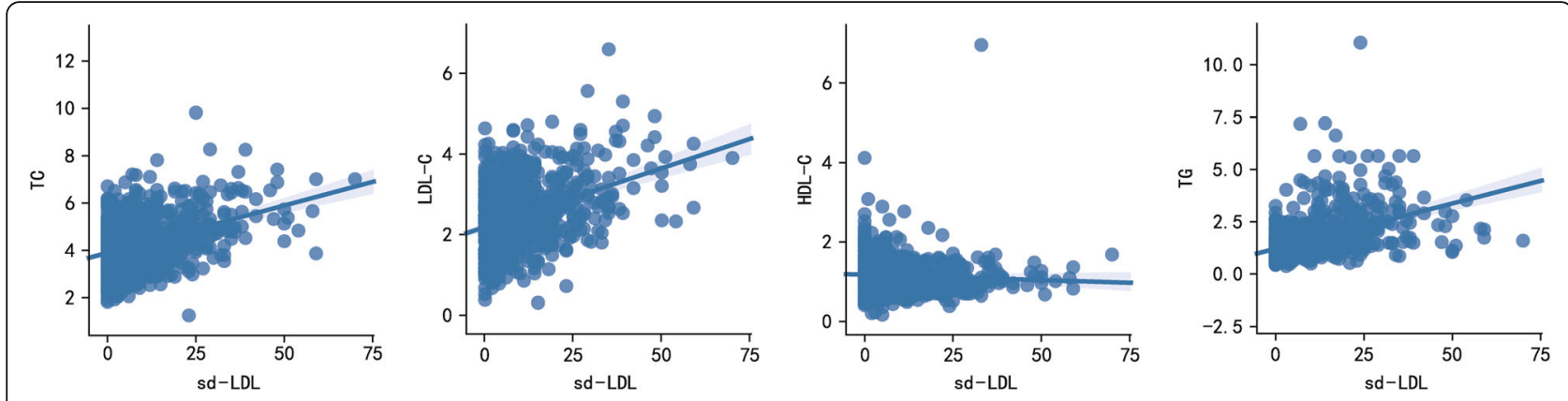

Fig. 2 Correlation between sd-LDL and serum lipids including TC, LDL-C, and TG

also showed that in CAD and metabolic syndrome patients, TG and TC were the most important determinants of serum sd-LDL concentrations [19]. Consequently, correlation coefficient between sd-LDL and serum lipids were analyzed. As a result, there is a significant positive correlation between sd-LDL levels and serum lipids including TC, LDL-C, and TG, which is consistent with findings reported by other groups.

LDL-C elevation has long been identified as a risk factor for AIS, however, AIS still occurs in people with normal LDL-C, suggesting it is not a sufficient biomarker for AIS prediction. Indeed, our data illustrated that sd-LDL levels in the high LDL-C group of AIS patients was higher than that in the normal group $(14.81 \pm 13.15$ vs. $8.03 \pm 8.71, P<0.001)$. Moreover, the levels of sd-LDL differed significantly between the AIS group and the control group when the level of LDL-C is normal $(P<0.05)$. Intriguingly, large LDL particles (LDL-1 and LDL-2) were significantly lower while LDL-3 and LDL-4, the main atherogenic particles of sd-LDL, were significantly higher in the AIS group. In addition, the other laboratory characteristics, including blood pressure, Scr, TC, HDL-C, TG, ApoA and ApoB also differed significantly between the two groups, indicating that the LDL-C levels alone does not accurately reflect risk to develop AIS. Studies in 22 countries have shown that hypertension is the main risk factor for stroke patients [20]. A high Scr concentration within the normal range is a marker for increased risk of cerebrovascular disease in both normotensive and hypertensive subjects [21]. Taken together, these observations may suggest that in patients with normal LDL-C levels, we need to consider other factors such

Table 5 Comparison of sd-LDL between the normal LDL-C group (LDL-C $<2.59 \mathrm{mmol} / \mathrm{L}$ ) and high LDL-C (LDL-C $\geq 2.59$ $\mathrm{mmol} / \mathrm{L}$ ) group of patients with AIS

\begin{tabular}{lccc}
\hline & Normal $(\boldsymbol{n}=\mathbf{3 1 4})$ & High $(\boldsymbol{n}=\mathbf{2 5 2})$ & $\boldsymbol{P}$ value * \\
\hline $\mathrm{sdLDL}(\mathrm{mg} / \mathrm{dL})$ & $5(2,11.2)$ & $11.5(4.8,23)$ & $<0.001^{*}$ \\
\hline
\end{tabular}

as blood pressure, Scr, and LDL subtractions to determine the risk of AIS.

Since even in those with normal LDL-C levels, the risk of AIS still exists and impacts of other features cannot be ruled out, a prediction model including most of the risk factors should be established for better prevention of the disease and reducing the associated burden. On the basis of previous studies and the data of this study, considering that it can be widely used in patients, especially in patients with normal LDL-C but still with high risk of AIS, we established a new prediction model including 12 stroke risk factors, namely Scr, SBP, DBP, TG, TC, LDL-C, HDL-C, LDL-1, LDL-2, LDL-3, LDL-4 and LDL-5. The prediction abilities of the four models

Table 6 Comparison of laboratory characteristics between the AIS group and control group with normal LDL-C levels $(<2.59$ $\mathrm{mmol} / \mathrm{L}$ )

\begin{tabular}{|c|c|c|c|}
\hline Characteristics & AIS $(n=314)$ & Controls $(n=127)$ & $P$ value * \\
\hline $\mathrm{SBP}(\mathrm{mmHg})$ & $137.5(127.3,154)$ & $123(123,135.5)$ & $<0.001 *$ \\
\hline $\mathrm{DBP}(\mathrm{mmHg})$ & $84(77,93)$ & $78(67.5,86)$ & $<0.001 *$ \\
\hline $\mathrm{GLU}(\mathrm{mmol} / \mathrm{L})$ & $5.2(4.6,6.2)$ & $5.2(4.6,6.4)$ & 0.356 \\
\hline $\mathrm{SCr}(\mu \mathrm{mol} / \mathrm{L})$ & $68.7(56,83.4)$ & $63(53,74.5)$ & $0.028 *$ \\
\hline $\mathrm{TC}(\mathrm{mmol} / \mathrm{L})$ & $3.5(3.1,4)$ & $3.7(3.3,4.2)$ & $0.016 *$ \\
\hline LDL-C (mmol/L) & $1.9(1.6,2.3)$ & $1.9(1.6,2.3)$ & 0.337 \\
\hline $\mathrm{HDL}-\mathrm{C}(\mathrm{mmol} / \mathrm{L})$ & $0.9(0.7,1.2)$ & $1.1(0.9,1.6)$ & $<0.001 *$ \\
\hline TG $(\mathrm{mmol} / \mathrm{L})$ & $1.3(1,1.8)$ & $1.1(0.9,1.4)$ & $<0.001 *$ \\
\hline ApoA (mmol/L) & $1.2(1.1,1.3)$ & $0.8(0.7,1)$ & $<0.001 *$ \\
\hline ApoB (mmol/L) & $0.6(0.5,0.8)$ & $0.7(0.6,0.7)$ & $<0.001 *$ \\
\hline sdLDL (mg/dL) & $5(2,11.2)$ & $3(1,5.5)$ & $<0.001 *$ \\
\hline LDL-1 (mg/dL) & $17(13,23)$ & $22(15,28)$ & $<0.001 *$ \\
\hline LDL-2 (mg/dL) & $15(10,20)$ & $16(11,21)$ & $0.043 *$ \\
\hline LDL-3 (mg/dL) & $5(2,9)$ & $3(1,5)$ & $<0.001 *$ \\
\hline LDL-4 (mg/dL) & $0(0,2)$ & $0(0,0)$ & $<0.001 *$ \\
\hline LDL-5 (mg/dL) & $0(0,0)$ & $0(0,0)$ & 0.065 \\
\hline LDL-6 (mg/dL) & $0(0,0)$ & $0(0,0)$ & 0.170 \\
\hline LDL-7 (mg/dL) & $0(0,0)$ & $0(0,0)$ & 0.185 \\
\hline
\end{tabular}


(a)

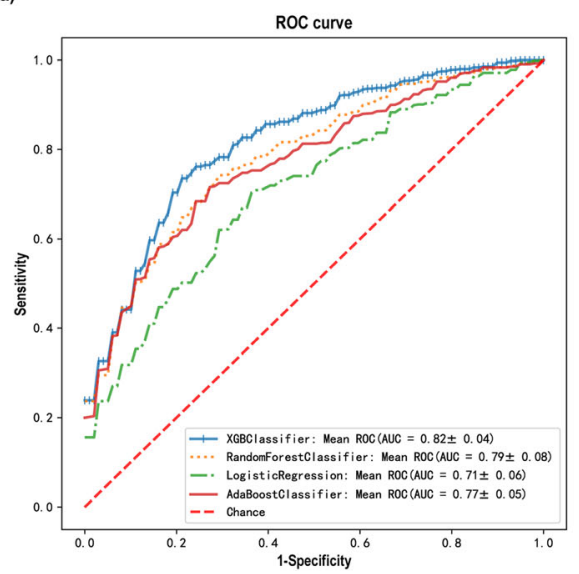

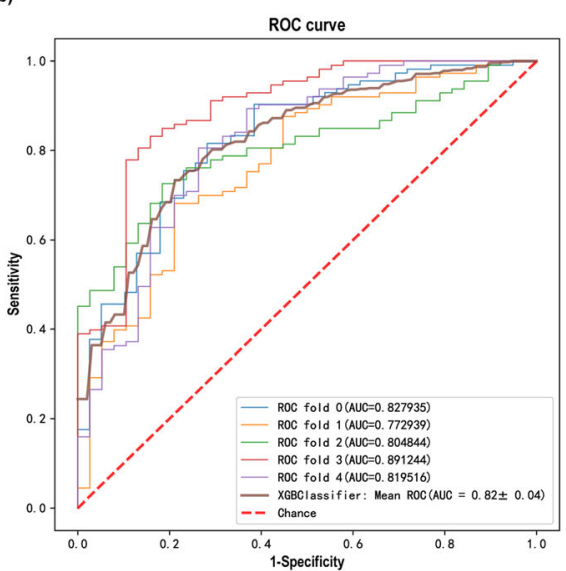

Fig. 3 Prediction of the relationship between laboratory characteristics and AIS with an established model. a Receiver operating characteristic (ROC) curve and area under the curve (AUC) for multiple classifiers including XGBoost, Random Forest, Logistic Regression, and AdaBoost. b The k-fold cross-validated ( $k=5)$ ROC curves for XGBoost classifier

of XGBoost, Random Forest, Logistic Regression, and AdaBoost classifier were assessed in this study. All models had AUC values above 0.70, and XGBoost achieved the best performance, exhibiting an average AUC of 0.82. It is generally considered that if the AUC of the model is greater than 0.70, the model has high accuracy [22]. The XGboost algorithm is a widely used machine learning method that can build complex models and make accurate decisions when given adequate data [23]. The accuracy and high efficiency of XGBoost make it show excellent performance in clinical research, especially in the field of vascular diseases [23-25]. The new prediction model can accurately predict the AIS risk in Chinese patients and all variables involved in the model can be simple and easily obtained on admission. The cut-off point was defined as 0.5 , and an output value $>0.5$ indicated AIS risk, which is easy for clinicians to accept and determine the most appropriate medical treatment for prevention.

\section{Conclusions}

In summary, our work analyzed the correlation of LDL subtractions with AIS. The results showed that LDL-3 and LDL-4 are positively correlated with AIS while LDL1 and LDL- 2 are negatively correlated with the disease even in the people with normal LDL-C levels. This finding indicates that levels of LDL subclasses should be considered in addition to serum LDL-C in assessment and management of AIS. Most importantly, a new prediction model including 12 variables were generated, which can help clinicians identify high-risk patients so that proper prevention measures can be taken to ease the potential burden and reduce suffering.

\section{Abbreviations}

AIS: Acute ischemic stroke; LDL: Low-density lipoproteins; GLU: Glucose; Scr: Serum creatinine; sd-LDL: Small dense LDL; TC: Total cholesterol; LDLC: Low density lipoprotein cholesterol; TG: Triglyceride; GBD: Global Burden of Disease; AS: Atherosclerosis; HDL: High-density lipoproteins; NCEP III: National Cholesterol Education Program Adult Treatment Panel III; CVD: Cardiovascular disease; CT: Computed tomography; MRI: Magnetic resonance imaging; HT: Hypertension; SBP: Systolic blood pressure; DBP: Diastolic blood pressure; DM: Diabetes mellitus; HDL-C: High density lipoprotein cholesterol; FDA: Food and Drug Administration; ROC: Receiver operating characteristics; AUC: Area under the curve; ApoA: Apolipoprotein A; ApoB: Apolipoprotein B; Al: Artificial intelligence; XGBoost: eXtreme gradient boosting; YLLs: Years of life lost; DALYs: Disability-adjusted life-years; sdLDL-C: Small dense Low density lipoprotein cholesterol; CAG: Coronary angiography; ox-LDL: Oxidized-low density lipoprotein

\section{Acknowledgements}

Not applicable.

\section{Authors' contributions}

$\mathrm{RD}, \mathrm{WX}, \mathrm{KW}, \mathrm{PM}$ and LD designed the study, oversaw the progress of the study, and wrote the manuscript. NY, HH and $\mathrm{HC}$ assisted with participant recruitment and provided methodological expertise. LW conceived and helped to design the study, assisting with data analysis and interpretation. All authors contributed significantly to the preparation and subsequent revisions of the manuscript. All authors have read and approved the final version of the manuscript.

\section{Funding}

This work was supported by Shanghai's industrial transformation and upgrading development project for absorption and innovation of imported technology (18XI-15) and Innovation Action Plan (18DZ2294600).

\section{Availability of data and materials}

All data generated or analyzed during this study are included in this published article.

\section{Ethics approval and consent to participate}

The study was carried out in accordance with The Code of Ethics of the World Medical Association (Declaration of Helsinki) for experiments involving humans, and the protocol was approved by the Ethics Committee of Hebei General Hospital, the First People's Hospital of Pingdingshan and the Affiliated Hospital of Chengde Medical University. Informed consent was obtained for experimentation with human subjects. Every individual was informed about the aims of the study and provided written consent before 
participation. Written informed consent was obtained from a relative in the case of patients with impaired cognition.

\section{Consent for publication}

Not applicable.

\section{Competing interests}

The authors declare that they have no competing interests.

\section{Author details}

${ }^{1}$ Department of Neurology, Hebei General Hospital, Shijiazhuang 050000

Hebei, China. ${ }^{2}$ Department of Neurology, the First People's Hospital of Pingdingshan, Henan 467000 Pingdingshan, China. ${ }^{3}$ Department of Neurosurgery, the Affiliated Hospital of Chengde Medical University, Chengde 067000, Hebei, China. ${ }^{4}$ Department of Medicine, Shanghai Zhangjiang institute of Medical innovation, Biotecan Pharmaceuticals co., Itd., Shanghai 201204, China.

Received: 24 August 2020 Accepted: 30 October 2020

Published online: 13 November 2020

\section{References}

1. Collaborators GBDLRoS, Feigin VL, Nguyen G, Cercy K, Johnson CO, Alam T, et al. Global, Regional, and Country-Specific Lifetime Risks of Stroke, 1990 and 2016. N Engl J Med. 2018;379(25):2429-37. https://doi.org/10.1056/ NEJMoa1804492

2. Kirshner HS. Differentiating ischemic stroke subtypes: risk factors and secondary prevention. J Neurol Sci. 2009;279(1-2):1-8. https://doi.org/10. 1016/j.jns.2008.12.012.

3. Schulz UG, Rothwell PM. Differences in vascular risk factors between etiological subtypes of ischemic stroke: importance of population-based studies. Stroke. 2003;34(8):2050-9. https://doi.org/10.1161/01.STR. $0000079818.08343 .8 \mathrm{C}$.

4. Rizzo M, Pernice V, Frasheri A, Di Lorenzo G, Rini GB, Spinas GA, et al. Small, dense low-density lipoproteins (LDL) are predictors of cardio- and cerebrovascular events in subjects with the metabolic syndrome. Clin Endocrinol. 2009:70(6):870-5. https://doi.org/10.1111/j.1365-2265.2008.03407.x.

5. Huh HJ, Park BT, Lee SY, Kang ES, Park HD. Establishing reference intervals for LDL subfractions in a Korean population using the Lipoprint LDL system. Clin Chem Lab Med. 2013;51(8):e179-82. https://doi.org/10.1515/ cclm-2012-0737.

6. National Cholesterol Education Program Expert Panel on Detection E, Treatment of High Blood Cholesterol in A. Third Report of the National Cholesterol Education Program (NCEP) Expert Panel on Detection Evaluation, and Treatment of High Blood Cholesterol in Adults (Adult Treatment Panel III) final report. Circulation. 2002;106(25):3143-421.

7. Hoefner DM, Hodel SD, O'Brien JF, Branum EL, Sun D, Meissner I, et al. Development of a rapid, quantitative method for LDL subfractionation with use of the Quantimetrix Lipoprint LDL system. Clin Chem. 2001; 47(2):266-74.

8. Varady KA, Lamarche B. Lipoprint adequately estimates LDL size distribution, but not absolute size, versus polyacrylamide gradient gel electrophoresis. Lipids. 2011;46(12):1163-7. https://doi.org/10.1007/s11745-011-3611-8.

9. Zhou M, Wang H, Zeng $X$, Yin P, Zhu J, Chen W, et al. Mortality, morbidity, and risk factors in China and its provinces, 1990-2017: a systematic analysis for the global burden of disease study 2017. Lancet. 2019. https://doi.org/ 10.1016/S0140-6736(19)30427-1

10. Kwon SW, Yoon SJ, Kang TS, Kwon HM, Kim JH, Rhee J, et al. Significance of small dense low-density lipoprotein as a risk factor for coronary artery disease and acute coronary syndrome. Yonsei Med J. 2006;47(3):405-14. https://doi.org/10.3349/ymj.2006.47.3.405.

11. Koba S, Hirano T, Ito Y, Tsunoda F, Yokota Y, Ban Y, et al. Significance of small dense low-density lipoprotein-cholesterol concentrations in relation to the severity of coronary heart diseases. Atherosclerosis. 2006;189(1):206-14 https://doi.org/10.1016/j.atherosclerosis.2005.12.002

12. Rizzo M, Pernice V, Frasheri A, Berneis K. Atherogenic lipoprotein phenotype and LDL size and subclasses in patients with peripheral arterial disease. Atherosclerosis. 2008;197(1):237-41. https://doi.org/10.1016/j.atherosclerosis. 2007.03.034.

13. Sakai K, Koba S, Nakamura Y, Yokota Y, Tsunoda F, Shoji M, et al. Small dense low-density lipoprotein cholesterol is a promising biomarker for secondary prevention in older men with stable coronary artery disease. Geriatr Gerontol Int. 2018;18(6):965-72. https://doi.org/10.1111/ggi.13287.

14. Zhao X, Sun D, Xu RX, Guo YL, Zhu CG, Wu NQ, et al. Low-density lipoprotein-associated variables and the severity of coronary artery disease: an untreated Chinese cohort study. Biomarkers. 2018;23(7):647-53. https:// doi.org/10.1080/1354750X.2018.1474256.

15. Chaudhary R, Mathew D, Bliden K, Tantry US, Sharma T, Gesheff MG, et al. Low-density lipoprotein 4: a novel predictor of coronary artery disease severity. Curr Med Res Opin. 2017;33(11):1979-84. https://doi.org/10.1080/ 03007995.2017.1365052.

16. Zeljkovic A, Vekic J, Spasojevic-Kalimanovska V, Jelic-Ivanovic Z, Bogavac Stanojevic N, Gulan B, et al. LDL and HDL subclasses in acute ischemic stroke: prediction of risk and short-term mortality. Atherosclerosis. 2010; 210(2):548-54. https://doi.org/10.1016/j.atherosclerosis.2009.11.040.

17. Kayran Y, Yayla V, Cabalar M, Bajrami A, Karamanli Y, Gedikbasi A, et al. LDL subclasses in ischemic stroke: a risk factor? Noro Psikiyatr Ars. 2019;56(1):137. https://doi.org/10.5152/npa.2017.19393.

18. Swinkels DW, Demacker PN, Hak-Lemmers HL, Mol MJ, Yap SH, van't Laar A Some metabolic characteristics of low-density lipoprotein subfractions, LDL1 and LDL-2: in vitro and in vivo studies. Biochim Biophys Acta. 1988;960(1): 1-9. https://doi.org/10.1016/0005-2760(88)90002-1.

19. Yazdandoust S, Parizadeh SM, Moohebati M, Yaghmaei P, Rahsepar AA, Tavallaie S, et al. Serum small dense low-density lipoprotein concentrations are elevated in patients with significant coronary artery stenosis and are related to features of the metabolic syndrome. Lipids. 2012;47(10):963-72. https://doi.org/10.1007/s11745-012-3706-x

20. O'Donnell MJ, Xavier D, Liu L, Zhang H, Chin SL, Rao-Melacini P, et al. Risk factors for ischaemic and intracerebral haemorrhagic stroke in 22 countries (the INTERSTROKE study): a case-control study. Lancet. 2010;376(9735):11223. https://doi.org/10.1016/S0140-6736(10)60834-3.

21. Wannamethee SG, Shaper AG, Perry IJ. Serum creatinine concentration and risk of cardiovascular disease: a possible marker for increased risk of stroke. Stroke. 1997;28(3):557-63. https://doi.org/10.1161/01.str.28.3.557.

22. Luo X, Lin F, Zhu S, Yu M, Zhang Z, Meng L, et al. Mine landslide susceptibility assessment using IVM, ANN and SVM models considering the contribution of affecting factors. PLoS One. 2019;14(4):e0215134. https://doi. org/10.1371/journal.pone.0215134.

23. Xiao J, Ding R, Xu X, Guan H, Feng X, Sun T, et al. Comparison and development of machine learning tools in the prediction of chronic kidney disease progression. J Transl Med. 2019;17(1):119. https://doi.org/10.1186/ s12967-019-1860-0.

24. Kilic A, Goyal A, Miller JK, Gleason TG, Dubrawksi A. Performance of a machine learning algorithm in predicting outcomes of aortic valve replacement. Ann Thorac Surg. 2020. https://doi.org/10.1016/j.athoracsur. 2020.05.107

25. Yu B, Qiu W, Chen C, Ma A, Jiang J, Zhou H, et al. SubMito-XGBoost: predicting protein submitochondrial localization by fusing multiple feature information and extreme gradient boosting. Bioinformatics. 2020;36(4): 1074-81. https://doi.org/10.1093/bioinformatics/btz734.

\section{Publisher's Note}

Springer Nature remains neutral with regard to jurisdictional claims in published maps and institutional affiliations.

Ready to submit your research? Choose BMC and benefit from

- fast, convenient online submission

- thorough peer review by experienced researchers in your field

- rapid publication on acceptance

- support for research data, including large and complex data types

- gold Open Access which fosters wider collaboration and increased citations

- maximum visibility for your research: over $100 \mathrm{M}$ website views per year

At $\mathrm{BMC}$, research is always in progress.

Learn more biomedcentral.com/submissions 